\title{
Strategic Energy Planning under Uncertainty: a Mixed- Integer Linear Programming Modeling Framework for Large-Scale Energy Systems
}

\author{
Stefano Moret ${ }^{\mathrm{a},}$, , Michel Bierlaire ${ }^{\mathrm{b}}$ and François Maréchal ${ }^{\mathrm{a}}$ \\ ${ }^{\mathrm{a}}$ Industrial Process and Energy Systems Engineering (IPESE) \\ ${ }^{\mathrm{b}}$ Transport and Mobility Laboratory \\ École Polytechnique Fédérale de Lausanne, 1015 Lausanne, Switzerland \\ *stefano.moret@epfl.ch
}

\begin{abstract}
Various countries and communities are defining strategic energy plans driven by concerns related to climate change and security of energy supply. Energy models are needed to support this decision-making process. The long time horizon inherent to strategic energy planning requires uncertainty to be accounted for. Most energy models available today are too complex or computationally expensive for uncertainty analyses to be carried out.

This study proposes a concise multi-period Mixed-Integer Linear Programming (MILP) formulation for strategic energy planning under uncertainty. The modeling framework allows optimizing the energy system in a snapshot future year having as objective the total annual cost and assessing as well the global $\mathrm{CO}_{2}$-equivalent emissions. Key features of the model are a clear distinction both between demand and supply and between resources and technologies, a low computational time and a multiperiod resolution to account for issues related to seasonality and energy storage. The model is applied to a real case study and a Global Sensitivity Analysis (GSA) highlights the impact of uncertain parameters in energy planning.
\end{abstract}

Keywords: strategic energy planning, energy modeling, Mixed-Integer Linear Programming (MILP), uncertainty, Global Sensitivity Analysis (GSA)

\section{Introduction}

Fossil fuels accounted for $81.7 \%$ of global primary energy supply in 2012 (IEA (2014)) and are the major cause of anthropogenic greenhouse gas emissions (IPCC (2011)). Various countries and communities are defining long-term energy plans to gradually replace fossil technologies with renewables and more efficient alternatives. This has been identified as a key priority in order to tackle the challenges of climate change and security of energy supply (IPCC (2011)). Strategic energy planning for large-scale energy systems involves investment decisions for energy conversion technologies over a time horizon of 20 to 50 years. Long-term energy forecasting models are inevitably inaccurate (Koomey et al. (2003)), especially when it comes to fuel price forecasts (Marnay and Siddiqui (2006)). Thus, taking into account uncertainty in energy modeling and planning is a priority.

Despite the importance of accounting for uncertainty, the penetration of uncertainty analysis in the energy field is still low. Grossmann et al. (2015) have identified in model complexity and high computational time the main barriers to its wider diffusion. Optimization is an effective tool for strategic planning of energy systems (Zeng et al. (2011)). MILP models in particular are widely 
used for this purpose (N.E. Koltsaklis et al. (2013)). Most of these models though focus only on one aspect (usually the electricity sector) and on the long-term evolution of the energy system without accounting for other sectors and for seasonal variations within single years.

The proposed MILP formulation overcomes these gaps by modeling the entire energy system and optimizing it in a "snapshot" future year. The multiperiod resolution allows taking into account aspects related to seasonality and energy storage. The concise formulation and low computational time make it suitable for optimization under uncertainty applications. Firstly the MILP formulation is presented in detail, then the model is applied to the real case study of Switzerland to highlight the impact of uncertain parameters in strategic energy planning.

\section{MILP model formulation}

According to the classification of energy models by Codina Gironès et al. (2015) the presented modeling framework belongs to the "snapshot" category. The main assumption is that the energy system is entirely rebuilt in a target year considering fuel prices and technology development status relative to that year. In this way, the cost of the energy transition is not accounted for.

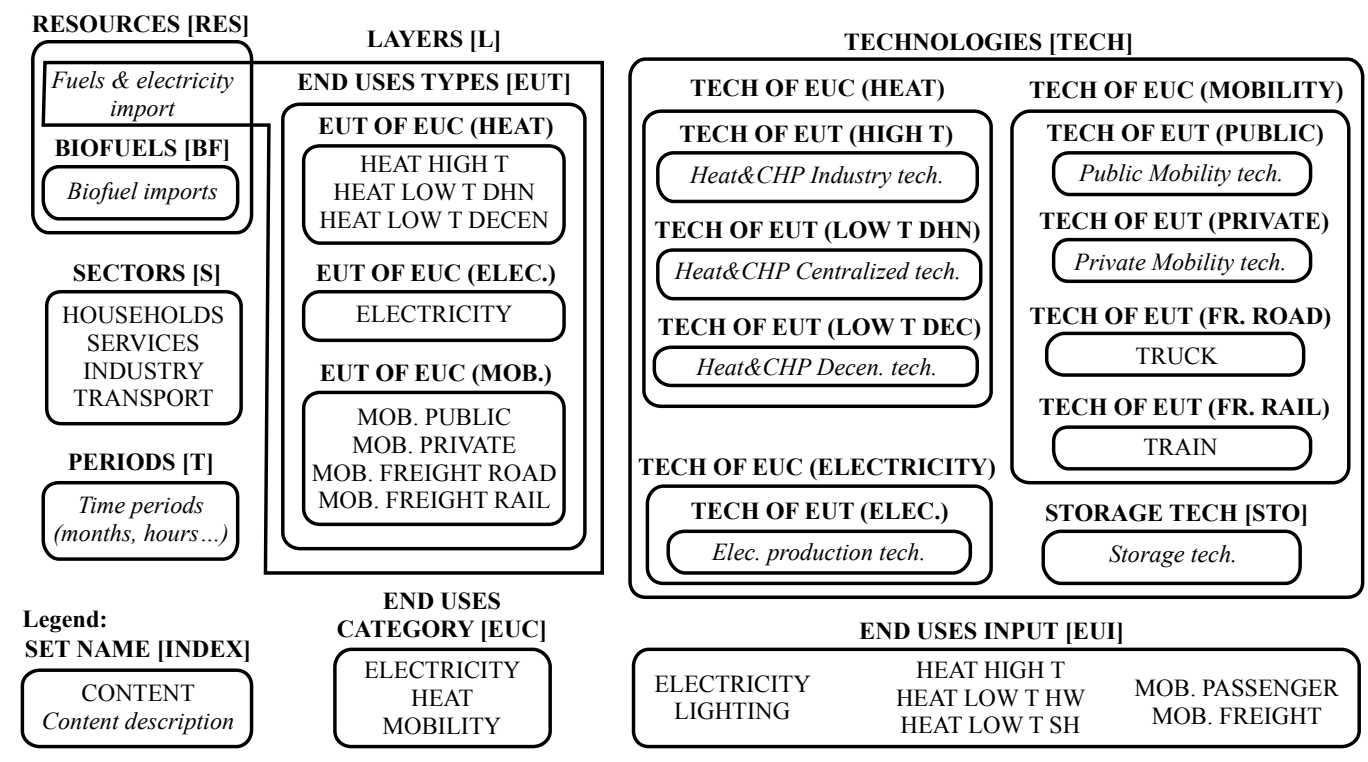

Figure 1: Sets of the MILP model

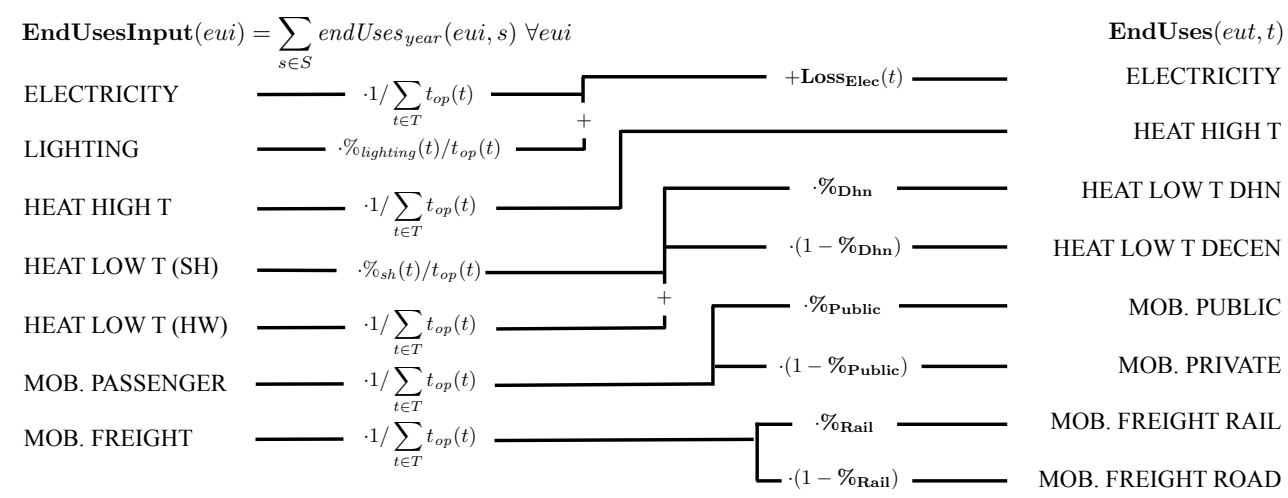

Figure 2: EndUses calculation starting from yearly demand model input (EndUsesInput) 
Table 1: Parameter list with description. Set indices as in Figure 1

\begin{tabular}{|c|c|c|}
\hline Parameter & Units & Description \\
\hline endUses $s_{\text {year }}($ eui,$s)$ & {$[\mathrm{GWh} / \mathrm{y}]^{a}$} & Annual end-uses in energy services per sector \\
\hline$i_{\text {rate }}$ & {$[-]$} & Real discount rate \\
\hline$\%_{\text {public,min }}, \%_{\text {public, } \max }$ & {$[-]$} & Upper and lower limit to \% Public \\
\hline$\%_{\text {rail,min }}, \%_{\text {rail,max }}$ & {$[-]$} & Upper and lower limit to $\%_{\text {Rail }}$ \\
\hline$\%_{d h n, \min }, \%_{d h n, \max }$ & {$[-]$} & Upper and lower limit to $\%_{\mathbf{D h n}}$ \\
\hline$t_{o p}(t)$ & [h] & Time periods duration \\
\hline$\%_{\text {lighting }}(t)$ & {$[-]$} & Yearly share (adding up to 1) of lighting end-uses \\
\hline$\%_{s h}(t)$ & {$[-]$} & Yearly share (adding up to 1 ) of SH end-uses \\
\hline$f($ res $\cup$ tech $\backslash$ sto,$l)$ & {$[\mathrm{GW}]^{b}$} & $\begin{array}{l}\text { Input from }(<0) \text { or output to }(>0) \text { layers. } f(i, j)=1 \\
\text { if } j \text { is main output layer for technology/resource } i\end{array}$ \\
\hline mult $_{r e f}($ tech $)$ & {$[\mathrm{GW}]^{b c}$} & Reference size with respect to main output \\
\hline$c_{i n v}($ tech $)$ & {$[\mathrm{M} € / \mathrm{GW}]^{b c}$} & Technology specific investment cost \\
\hline $\begin{array}{l}c_{\text {maint }}(\text { tech }) \\
n(\text { tech })\end{array}$ & {$[\mathrm{M} \in / \mathrm{GW} / \mathrm{y}]^{b c}$} & Technology specific yearly maintenance cost \\
\hline$f_{\min }, f_{\max }($ tech $)$ & {$[\mathrm{GW}]^{b c}$} & $\begin{array}{l}\text { Technology lifetime } \\
\text { Min./max. installed size of the technology }\end{array}$ \\
\hline avail(res) & {$[\mathrm{GWh} / \mathrm{y}]$} & Resource yearly total availability \\
\hline$c_{p, t}($ tech,$t)$ & {$[-]$} & Period capacity factor (default 1 ) \\
\hline$c_{p}(t e c h)$ & {$[-]$} & Yearly capacity factor \\
\hline$c_{o p}($ res,$t)$ & {$[\mathrm{M} \in / \mathrm{GWh}]$} & Specific cost of resources \\
\hline$\varepsilon_{\text {sto, in }}, \varepsilon_{\text {sto,out }}($ sto,$l)$ & {$[-]$} & $\begin{array}{l}\text { Efficiency }[0 ; 1] \text { of storage input from/output } \\
\text { to layer. Set to } 0 \text { if storage not related to layer. }\end{array}$ \\
\hline$\%_{\text {loss }_{\text {Elec }}}$ & {$[-]$} & Losses $[0 ; 1]$ in the electricity grid \\
\hline
\end{tabular}

Table 2: Variable list with description. All variables are continuous and non-negative, unless otherwise indicated.

\begin{tabular}{|c|c|c|}
\hline Variable & Units & Description \\
\hline EndUsesInput $($ eui $)$ & {$[\mathrm{GWh} / \mathrm{y}]^{a}$} & Total annual end-uses in energy services \\
\hline $\operatorname{EndUses}(l, t)$ & {$[\mathrm{GW}]^{b}$} & End-uses demand. Set to 0 if $l \notin E U T$ \\
\hline$\%$ Public & {$[-]$} & Ratio $[0 ; 1]$ public mobility over total passenger mobility \\
\hline$\%_{\text {Rail }}$ & [-] & Ratio $[0 ; 1]$ rail transport over total freight transport \\
\hline \% Dhn & {$[-]$} & Ratio $[0 ; 1]$ centralized over total low-temperature heat \\
\hline $\mathbf{N}($ tech $) \in \mathbb{N}$ & {$[-]$} & Number of installed units of size mult $_{r e f}$ \\
\hline Mult $(t e c h)$ & {$[\mathrm{GW}]^{b c}$} & Installed capacity with respect to main output \\
\hline $\mathbf{M u l t}_{\mathbf{t}}($ tech $\cup \operatorname{res}, t)$ & {$[\mathrm{GW}]^{b c}$} & Operation in each period \\
\hline $\mathbf{C}_{\mathbf{i n v}}($ tech $)$ & {$[\mathrm{M} €]$} & Technology total investment cost \\
\hline $\mathbf{C}_{\text {maint }}($ tech $)$ & {$[\mathrm{M} € / \mathrm{y}]$} & Technology yearly maintenance cost \\
\hline $\mathbf{C}_{\mathbf{o p}}($ res $)$ & {$[\mathrm{M} € / \mathrm{y}]$} & Total cost of resources \\
\hline$\tau($ tech $)$ & {$[-]$} & Investment cost annualization factor \\
\hline $\mathbf{S t o}_{\text {in }}$, Sto $_{\text {out }}($ sto $, l, t)$ & {$[\mathrm{GW}]$} & Input to/output from storage units \\
\hline $\operatorname{LosS}_{\text {Elec }}(t)$ & {$[\mathrm{GW}]$} & Electricity transmission losses \\
\hline
\end{tabular}

${ }^{a}[\mathrm{Mpkm}]$ (millions of passenger-km) for passenger, [Mtkm] (millions of ton-km) for freight mobility end-uses

${ }^{b}[\mathrm{Mpkm} / \mathrm{h}]$ for passenger, [Mtkm/h] for freight mobility end-uses

${ }^{c}[\mathrm{GWh}]$ if tech $\in$ STO

Figure 1 shows the sets and subsets of the optimization model with their relative indices used throughout the paper, while Table 1 and Table 2 list and describe the parameters and variables, respectively. Units should be proportionally scaled based on the specific application.

Figure 2 shows the constraints relative to the calculation of the end-uses demand in each period EndUses starting from the projected total yearly demand (EndUsesInput, input from demand- 
side model) summed across the different energy sectors (households, services, industry, transport). Electricity end-uses result from the sum of the electricity-only demand, assumed constant throughout the year, and the demand for lighting, distributed across the periods according to \% lighting. Low-temperature heat demand results from the sum of yearly demand for hot water $(\mathrm{HW})$, evenly shared across the year, and space heating $(\mathrm{SH})$, distributed across the periods according to $\%_{s h}$. The percentage repartition between centralized (DHN) and decentralized heat demand is defined by the variable $\%$ Dhn. High temperature process heat and mobility demand are evenly distributed across the periods. The variables $\%$ Public and $\%$ Rail define the penetration of public transportation in passenger mobility and of train in freight, respectively.

The optimization problem is defined as follows:

$$
\begin{aligned}
& \min \sum_{j \in T E C H}\left(\tau(j) \mathbf{C}_{\mathbf{i n v}}(j)+\mathbf{C}_{\text {maint }}(j)\right)+\sum_{i \in R E S} \mathbf{C}_{\mathbf{o p}}(i) \\
& \text { s.t. } \tau(j)=\frac{i_{\text {rate }}\left(i_{\text {rate }}+1\right)^{n(j)}}{\left(i_{\text {rate }}+1\right)^{n(j)}-1} \\
& \mathbf{C}_{\text {inv }}(j)=c_{i n v}(j) * \operatorname{Mult}(j) \\
& \mathbf{C}_{\text {maint }}(j)=c_{\text {maint }}(j) * \operatorname{Mult}(j) \\
& f_{\text {min }}(j) \leq \operatorname{Mult}(j) \leq f_{\max }(j) \\
& \mathbf{N}(j) \text { mult }_{\text {ref }}(j)=\mathbf{M u l t}(j) \\
& \operatorname{Mult}_{\mathbf{t}}(j, t) \leq \operatorname{Mult}(j) c_{p, t}(j, t) \\
& \sum_{t \in T} \operatorname{Mult}_{\mathbf{t}}(j, t) t_{o p}(t) \leq \operatorname{Mult}(j) c_{p}(j) \sum_{t \in T} t_{o p}(t) \\
& \mathbf{C}_{\mathbf{o p}}(i)=\sum_{t \in T} c_{o p}(i, t) \mathbf{M u l t}_{\mathbf{t}}(i, t) t_{o p}(t) \\
& \sum_{t \in T} \mathbf{M u l t}_{\mathbf{t}}(i, t) t_{o p}(t) \leq \operatorname{avail}(i) \\
& \sum_{i \in R E S \cup T E C H \backslash S T O} f(i, l) \mathbf{M u l t}_{\mathbf{t}}(i, t)+\sum_{j \in S T O}\left(\operatorname{Sto}_{\text {out }}(j, l, t)-\mathbf{S t o}_{\text {in }}(j, l, t)\right)-\operatorname{EndUses}(l, t)=0 \\
& \forall l \in L, \forall t \in T \\
& \forall j \in T E C H \text { (2) } \\
& \forall j \in T E C H \text { (3) } \\
& \forall j \in T E C H \text { (4) } \\
& \forall j \in T E C H \text { (5) } \\
& \forall j \in T E C H \text { (6) } \\
& \forall j \in T E C H, \forall t \in T \text { (7) } \\
& \forall j \in T E C H \text { (8) } \\
& \forall i \in R E S \text { (9) } \\
& \forall i \in R E S(10) \\
& \operatorname{Mult}_{\mathbf{t}}(j, t)=\mathbf{M u l t}_{\mathbf{t}}(j, t-1)+t_{o p}(t) \text {. }
\end{aligned}
$$

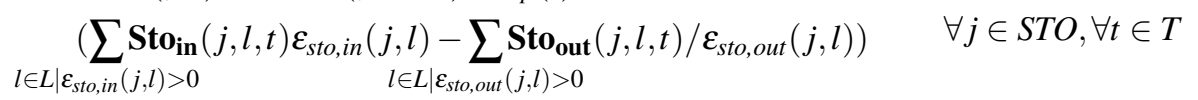

$$
\begin{aligned}
& \operatorname{Sto}_{\text {in }}(j, l, t)\left(\left\lceil\varepsilon_{\text {sto,in }}(j, l)\right\rceil-1\right)=0 \quad \forall j \in S T O, \forall l \in L, \forall t \in T \\
& \operatorname{Sto}_{\text {out }}(j, l, t)\left(\left\lceil\varepsilon_{\text {sto,out }}(j, l)\right\rceil-1\right)=0 \quad \forall j \in S T O, \forall l \in L, \forall t \in T
\end{aligned}
$$

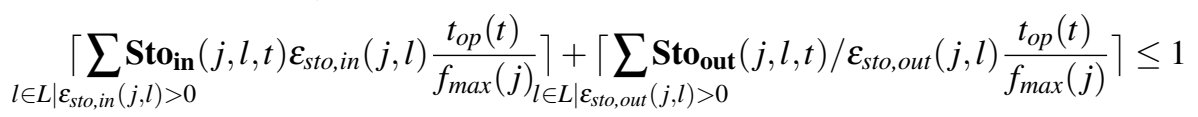

$$
\begin{aligned}
& \forall j \in S T O, \forall t \in T \text { (15) }
\end{aligned}
$$

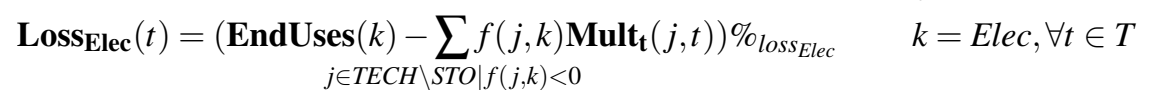

$$
\begin{aligned}
& \operatorname{Mult}_{\mathbf{t}}(j, t)+\text { Mult }_{\mathbf{t}}(k, t) \geq \\
& \frac{\text { EndUses }(H e a t L o w T D H N, t)+\text { EndUses }(H e a t L o w T D e c, t)}{\text { EndUsesInput }(H e a t L o w T S H)+\text { EndUsesInput }(\text { HeatLowTHW })} \sum_{t \in T} \operatorname{Mult}_{\mathbf{t}}(j, t) t_{o p}(t) \\
& \left.k=\text { Dec }_{\text {Solar }}, \forall j \in \text { TECH OF EUT(HeatLowTDec }\right) \backslash\{k\}, \forall t \in T \text { (17) }
\end{aligned}
$$


from the multiplication of its specific investment $\operatorname{cost} c_{i n v}$ and installed size Mult, the latter defined with respect to the relative end-uses category of output (Eq.(3)). $\mathbf{C}_{\mathbf{i n v}}$ is annualized with the factor $\tau$, calculated based on the interest rate $i_{\text {rate }}$ and the technology lifetime $n$ (Eq.(2)). The total maintenance cost is calculated in the same way (Eq.(4)). Upper and lower limits to the installed capacity of each technology are set by $f_{\max }$ and $f_{\min }$. The latter allows accounting for old technologies still existing in the "snapshot" year (Eq.(5)). Eq.(6) forces the number of installed units

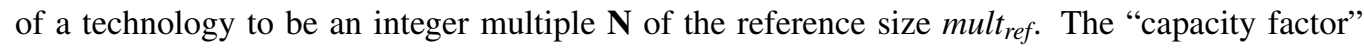
is conceptually divided into two components: a capacity factor for each period $c_{p, t}$ depending on resource availability (e.g. renewables) and a yearly capacity factor $c_{p}$ accounting for technology downtime and maintenance (Eq.(8)). Eq.(7) links the installed size of a technology to its actual use in each period Mult $\mathbf{t}_{\mathbf{t}}$ via the period capacity factor. The total cost of imported resources is calculated as the sum of the use over different periods multiplied by the period duration $t_{o p}$ and the specific cost of the resource $c_{o p}$ (Eq.(9)). The total use of resources is limited by the yearly availability avail (Eq.(10)).

Layers are defined as all the elements in the system that need to be balanced in each period, such as resources and end-uses demand. The matrix $f$ defines for all technologies and resources outputs to (positive) and inputs from (negative) layers. Eq.(11) expresses the balance for each layer: all outputs from resources and technologies (including storage) are used to satisfy end-uses demand or as inputs to other resources and technologies. Storage technologies allow storage across different periods and layers. The storage is modeled as a "tank" whose level (Mult $\mathbf{t})$ in period $t$ is equal to the level at the end of the previous period plus input to the storage Sto in minus output Sto ${ }_{\text {out }}$ in $t$ (Eq.(12)). The parameters $\varepsilon_{\text {sto, in }}$ and $\varepsilon_{\text {sto,out }}$ define efficiencies for storage input and output, respectively: if the efficiency is 0 then the storage technology and the layer are incompatible (Eq.(13)-(14)). Eq.(15), displayed in a compact non-linear formulation, ensures that the storage is not used as a transfer unit within a given period. Losses in the electricity grid Loss Elec $_{\text {are calcu- }}$ lated as a percentage $\%_{\text {loss }_{\text {Elec }}}$ of the total electricity needs in the system (Eq.(16)). Eq.(17) makes the model more realistic by defining the operating strategy for decentralized heating: the relative use of each technology in each period should be constant, except for solar thermal (Dec Solar $\left._{1}\right)$ which is given priority for operation.

The separation between resources and technologies allows an easy calculation of the global $\mathrm{CO}_{2}$ equivalent emissions by defining a set of constraints symmetrical to the ones used for the total cost. In this framework, operating emissions related to fuels (from cradle to combustion) are allocated to resources, while construction, maintenance and end-of-life emissions are assigned to technologies and annualized based on their lifetime.

\section{Global Sensitivity Analysis}

As an example, the model is applied to the energy system of Switzerland. In particular, this application of the model can be considered the MILP equivalent of the Microsoft Excel ${ }^{\mathrm{TM}}$ model presented in Codina Gironès et al. (2015), and currently implemented in the energy calculator of the online platform swiss-energyscope.ch (Moret et al. (2014)).

A GSA is performed in order to evaluate the impact of uncertain parameters on energy planning decisions. The example model features more than 500 uncertain parameters. A complete GSA would require characterizing the uncertainty of each parameter, which is out of the scope of this work. Thus, a $\pm 10 \%$ variation for all the uncertain parameters, grouped as in Table 1, is assumed. The GSA is applied with the Elementary Effects (EE) screening method (Morris (1991)). This method is effective in separating between influential and non-influential model uncertain parameters. Results are shown in Figure 3, plotting the mean $\mu$ and the standard deviation $\sigma$ of each parameter's EE onto the objective. As thoroughly described in Sin et al. (2009), parameters falling inside the red "V" in the plot are non-influential on the objective, while high absolute values of $\mu$ denote a relevant influence. In this application the most influential parameter is endUses year, followed by the efficiencies of technologies $(f)$ and the operating and investment costs. 


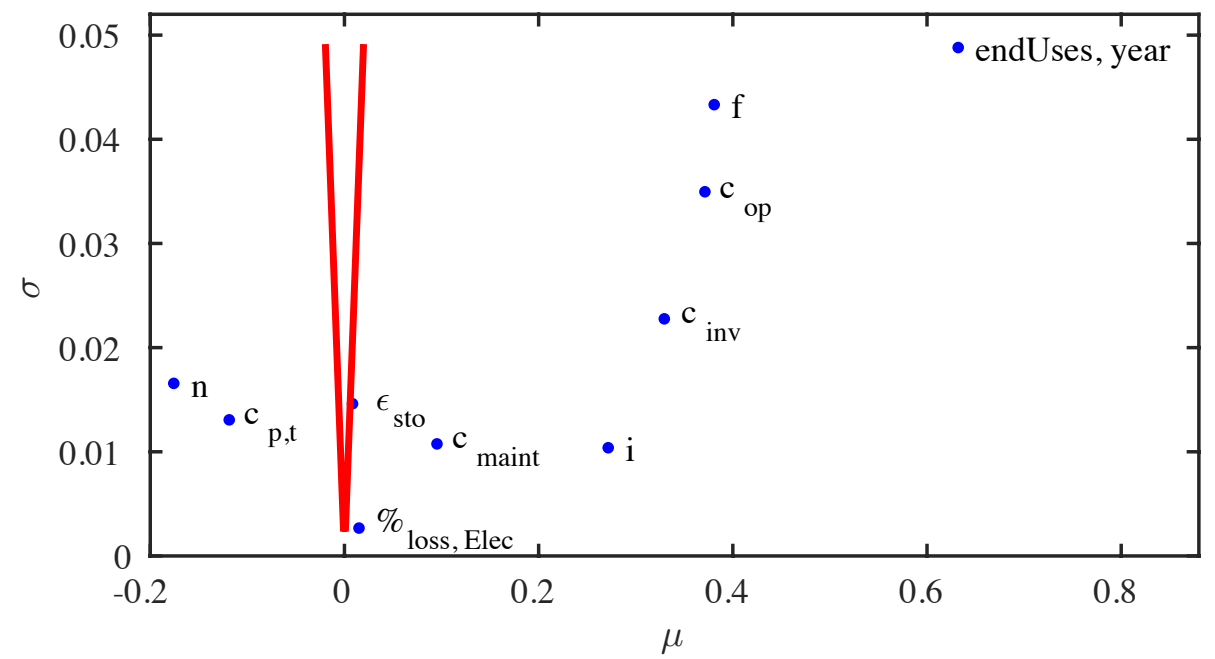

Figure 3: GSA: Elementary Effects of uncertain parameters onto the objective. The red "V" $(\mu=$ $\pm 2 S E M$, SEM: standard error of the mean) separates influential and non-influential parameters.

\section{Conclusions}

A MILP formulation for large-scale energy system planning is proposed. The model optimizes the total cost of the energy system in a "snapshot" future year assessing as well the $\mathrm{CO}_{2}$-equivalent emissions. The concise formulation and low computational time (less than 0.5 " for the example application) make it suitable for optimization under uncertainty applications. As an example, the model has been applied to a real case study and a GSA shows the relevant impact of uncertainty in energy planning decisions. Future work envisions the use of this formulation as a testbed for the definition of a methodology for uncertainty classification and optimization under uncertainty in energy planning applications.

\section{References}

V. Codina Gironès, S. Moret, F. Maréchal, D. Favrat, Oct. 2015. Strategic energy planning for large-scale energy systems: A modelling framework to aid decision-making. Energy 90, Part 1, 173-186.

I. E. Grossmann, R. M. Apap, B. A. Calfa, P. Garcia-Herreros, Q. Zhang, 2015. Recent Advances in Mathematical Programming Techniques for the Optimization of Process Systems under Uncertainty. In: J. K. H. a. R. G. Krist V. Gernaey (Ed.), Computer Aided Chemical Engineering. Vol. 37 of 12th International Symposium on Process Systems Engineering and 25th European Symposium on Computer Aided Process Engineering. Elsevier, pp. 1-14.

IEA, 2014. Key World Energy Statistics 2014. Tech. rep., IEA - International Energy Agency.

IPCC, 2011. Special Report on Renewable Energy Sources and Climate Change Mitigation. Cambridge University Press, United Kingdom and New York, NY, USA.

J. Koomey, P. Craig, A. Gadgil, D. Lorenzetti, 2003. Improving Long-Range Energy Modeling: A Plea for Historical Retrospectives. The Energy Journal 24 (4), 75-91.

C. Marnay, A. S. Siddiqui, 2006. Addressing an Uncertain Future Using Scenario Analysis. Tech. Rep. LBNL-62313, Ernest Orlando Lawrence Berkeley National Laboratory.

S. Moret, V. Codina Gironès, F. Maréchal, D. Favrat, 2014. Swiss-EnergyScope.ch: a Platform to Widely Spread Energy Literacy and Aid Decision-Making. Chemical Engineering Transactions 39, 877-882.

M. D. Morris, May 1991. Factorial Sampling Plans for Preliminary Computational Experiments. Technometrics 33 (2), 161-174.

N.E. Koltsaklis, A.S. Dagoumas, G.M. Kopanos, E.N. Pistikopoulos, M.C. Georgiadis, 2013. A Mathematical Programming Approach to the Optimal Long-Term National Energy Planning. In: Chemical Engineering Transactions.

G. Sin, K. V. Gernaey, A. E. Lantz, Jul. 2009. Good modeling practice for PAT applications: Propagation of input uncertainty and sensitivity analysis. Biotechnology Progress 25 (4), 1043-1053.

Y. Zeng, Y. Cai, G. Huang, J. Dai, Oct. 2011. A Review on Optimization Modeling of Energy Systems Planning and GHG Emission Mitigation under Uncertainty. Energies 4 (10), 1624-1656. 